\title{
Weight-driven growing networks
}

\author{
T. Anta* and P. L. Krapivsky \\ Center for Polymer Studies and Department of Physics, Boston University, Boston, MA 02215, USA
}

\begin{abstract}
We study growing networks in which each link carries a certain weight (randomly assigned at birth and fixed thereafter). The weight of a node is defined as the sum of the weights of the links attached to the node, and the network grows via the simplest weight-driven rule: A newly-added node is connected to an already existing node with the probability which is proportional to the weight of that node. We show that the node weight distribution $n(w)$ has a universal, that is independent on the link weight distribution, tail: $n(w) \sim w^{-3}$ as $w \rightarrow \infty$. Results are particularly neat for the exponential link weight distribution when $n(w)$ is algebraic over the entire weight range.
\end{abstract}

PACS numbers: 02.50.Cw, 05.40.-a, 05.50.+q, 87.18.Sn

\section{INTRODUCTION}

The network structure lies beneath many physical, biological, social, economical, and other real-world systems. Examples include resister networks, metabolic networks, communication networks like the Internet, information networks like the world-wide web, transportation networks, food webs, etc. Some networks are engineered while others like the world-wide web are created in a chaotic manner, viz. by the uncoordinated actions of many individuals, and yet they show a great deal of selforganization [1]. This surprising order of seemingly disordered networks was noticed long ago in the context of random graphs [2, 3], and it is also apparent in the models of growing random networks that have been thoroughly studied in the past few years (see reviews [4, 5, 6] and references therein).

Networks or graphs are defined as sets of nodes joined by links. A link in a graph merely indicates that a given pair of nodes is connected. If however connections differ in "strength", one may formalize this property by assigning a weight to each link. Many real-world networks are intrinsically weighted. In a collaboration network, the weight of a link between co-authors measures the strength of the collaboration such as the number of jointly-authored publications [7, 8]. In the airline transportation network, the weight of a link between two airports gives the passenger capacity on this route [9, 10]. Thus mathematically the weighted network is a graph in which each link carries a certain number which is called the weight. Weighted networks appear in literature under different names. For instance, the multigraph - that is the graph in which two nodes can be joined by multiple links - can be replaced by the graph in which a link between two nodes carries the integer weight equal to the number of links in the original multigraph joining those two nodes. Numerous engineering and mathematical studies of the flows in networks also treat weighted

* On leave from Institute for Theoretical Physics, Eötvös University, Budapest, Hungary

${ }^{\dagger}$ Electronic address: paulk@bu.edu networks - the weight (usually termed "capacity") represents the maximum allowed flow 11, 12]. Resistors networks (see e.g. [13, 14, 15]) form another very important class of weighted networks.

The models of weighted networks are usually close to the unweighted ones as far as the underlying graph structure is concerned. In other words, the link weights are passive variables. This allows to study weighted networks using the knowledge of unweighted networks as the starting point [16, 17]. In reality, the link weights can of course affect the graph structure. The range of possible models in which the link weights are active variables is extremely broad (see e.g. [18, 19, 20, 21, 22]) while the mechanisms driving the evolution of the real-world networks are still hardly known. In such situation one wants to study a minimal rather than detailed models. Here we introduce and investigate a minimal model of the weight-driven growth.

In Sec. III we introduce the minimal model precisely, and determine the node weight distribution and the joint node weight-degree distribution. We then compute the in-component weight distribution. In Sec. III we discuss some generalizations of the minimal model. We conclude in Sec. IV]

\section{THE MODEL}

The model is defined as follows. Each link carries a positive weight $w$ which is drawn from a certain distribution $\rho(w)$. We shall assume that the weights are positive 23]. The weight is assigned to the link when the link is created, and it remains fixed thereafter. The weight of a node is the sum of the weights of the links attached to the node. When a new node is added, it is linked to a single "target" node with probability proportional to the weight of the target node.

We assume that only one link emanates from each newly-introduced node, so the resulting network is a tree; the general case when a few links emanate from each node is discussed in Sec. III] The weight of a node (also termed as the node strength by some authors) increases when a new link is attached to it, yet, as the size of the 
network grows, the node weight distribution approaches a stationary distribution. Our first goal is to determine this distribution.

\section{A. Weight Distribution}

Let $N$ be the total number of nodes in the network and $N_{w}(N) d w$ the number of nodes whose weight lies in the range $(w, w+d w)$. When $N$ is large, it can be treated as a continuous variable, and $N_{w}(N)$ satisfies

$$
\frac{d N_{w}}{d N}=\frac{1}{W}\left[\int_{0}^{w} d x \rho(w-x) x N_{x}-w N_{w}\right]+\rho(w),
$$

where $W(N)=\int_{0}^{\infty} d w w N_{w}(N)$ is the total weight of all nodes [24]. The term in Eq. (11) which is proportional to $x N_{x} / W$ accounts for nodes with weight $x$ which gain a link of weight $w-x$ thereby creating nodes of weight $w$. The term $w N_{w} / W$ is the corresponding loss term. The last term accounts for the newly introduced node which has the same weight as its link.

$N_{w}(N)=N n(w)$ for large $N$, i.e. the weight distribution approaches a stationary ( $N$ independent) distribution $n(w)$. Hence, Eq. (11) simplifies to

$$
(\lambda+w) n(w)=\int_{0}^{w} d x \rho(w-x) x n(x)+\lambda \rho(w),
$$

where $\lambda=\int_{0}^{\infty} d w w n(w)$ is the average node weight. The total weight of all nodes is twice the total weight of all links (since each link connects two nodes) and the same obviously holds for the average weights. Therefore

$$
\lambda=2\langle w\rangle=2 \int_{0}^{\infty} d w w \rho(w) .
$$

We now specialize Eqs. (2) to the uniform link weight distribution

$$
\rho(w)=\left\{\begin{array}{ll}
1 & w<1 \\
0 & w>1
\end{array} .\right.
$$

Using relation $\lambda=2\langle w\rangle=1$ and the shorthand notation $F(w)=1+\int_{0}^{w} d x x n(x)$ we recast (2) into

$$
(1+w) n(w)= \begin{cases}F(w) & w<1 \\ F(w)-F(w-1) & w>1\end{cases}
$$

One can solve (4) recursively. Using $F^{\prime}(w)=w n(w)$ we find that for $w<1$ the cumulative distribution satisfies $\left[w^{-1}+1\right] F^{\prime}(w)=F(w)$ (here $F^{\prime}=d F / d w$ ). Solving this equation subject to the boundary condition $F(0)=1$ yields $F(w)=(1+w)^{-1} e^{w}$. Therefore the weight distribution is

$$
n(w)=(1+w)^{-2} e^{w} \quad \text { for } \quad w<1 .
$$

On the next interval $1<w<2$, we ought to solve

$$
\left(w^{-1}+1\right) \frac{d F}{d w}=F-w^{-1} e^{w-1} .
$$

The solution should match the previous one; this gives the boundary condition $F(1)=e / 2$. The resulting cumulative distribution is $F=e^{w-1}(e+1-w) /(1+w)$, from which we obtain the node weight distribution

$$
n(w)=\frac{e^{w-1}}{1+w}\left(\frac{e+1-w}{1+w}-\frac{1}{w}\right) \quad \text { for } \quad 1<w<2 .
$$

Interestingly, the weight distribution loses continuity at the cutoff value $w=1$ of the uniform link weight distribution: $n(1-0)=e / 4$ while $n(1+0)=(e-2) / 4$. Proceeding, one finds the node weight distribution on the interval $2<w<3$, etc. The distribution is analytic everywhere apart from the integer values; at the integer value $w=k \geq 2$, the node weight distribution is continuously differentiable $k-2$ times (see Fig. 1). The analytic expressions for the node weight distribution are very cumbersome for large $w$. Fortunately, the asymptotic behavior in the tail region is very simple. To extract the asymptotic we expand the right-hand side of (4) using the Taylor series:

$$
\begin{aligned}
F(w)-F(w-1) & =F^{\prime}(w)-\frac{1}{2} F^{\prime \prime}(w)+\ldots \\
& =w n(w)-\frac{1}{2}[w n(w)]^{\prime}+\ldots .
\end{aligned}
$$

Plugging this expansion into (4) we obtain

$$
w \frac{d n}{d w}+3 n=0,
$$

which is solved to give

$$
n(w) \rightarrow \frac{A}{w^{3}} \quad \text { when } \quad w \rightarrow \infty .
$$

The amplitude $A$ cannot be found by solving the linear equation (15); its determination requires analysis of the full problem (4).

Note also that for the deterministic link weight distribution $\rho(w)=\delta(w-1)$ the weight of the node is equal to the degree, so the model becomes equivalent to the basic growing network model with preferential attachment that also leads to a node weight distribution with $w^{-3}$ tail. In general, the large weight tail of the node weight distribution is always given by Eq. (6) if the link weight distribution has a cutoff. To prove this assertion suppose that $\rho(w)=0$ for $w>1$. For $w>1$, Eq. (2) becomes

$$
(\lambda+w) n(w)=\Phi(w)
$$

with $\Phi(w)=\int_{0}^{1} d x \rho(x)(w-x) n(w-x)$; for the uniform distribution $\Phi(w)=F(w)-F(w-1)$ and Eq. (7) turns into Eq. (4). Expanding the integrand in $\Phi(w)$

$$
(w-x) n(w-x)=w n(w)-x[w n(w)]^{\prime}+\ldots
$$




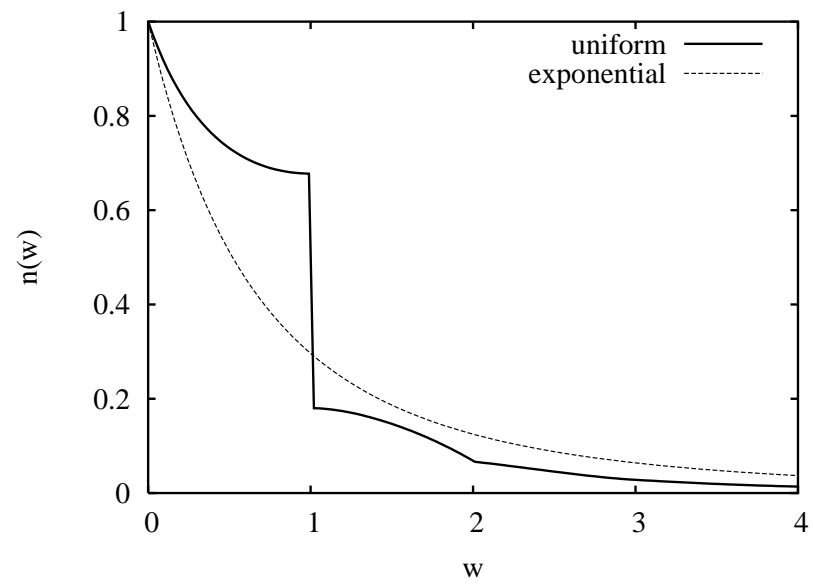

FIG. 1: The node weight distribution $n(w)$ emerging for uniform and exponential link weight distributions.

yields

$$
\Phi(w)=w n(w)-\frac{\lambda}{2}[w n(w)]^{\prime}+\ldots,
$$

where relations $\int d w \rho(w)=1$ and $\int d w w \rho(w)=\lambda / 2$ were used. In conjunction with (9), Eq. (7) reduces to Eq. (5) leading to the tail given by (6). This derivation, as well as the earlier one that led to Eq. (5), ignores the higher terms in the expansion (8). Keeping such terms one would obtain

$$
w \frac{d n}{d w}+3 n=\kappa_{2}[w n(w)]^{\prime \prime}-\frac{1}{3} \kappa_{3}[w n(w)]^{\prime \prime \prime}+\ldots
$$

with $\kappa_{2}=\lambda^{-1} \int d w w^{2} \rho(w), \kappa_{3}=\lambda^{-1} \int d w w^{3} \rho(w)$, etc. Solving (10) gives higher order corrections to the tail asymptotics

$$
n=\frac{A}{w^{3}}\left[1-\frac{6 \kappa_{2}}{w}+\frac{36\left(\kappa_{2}\right)^{2}-4 \kappa_{3}}{w^{2}}+\ldots\right]
$$

but it does not affect the leading $w^{-3}$ tail.

The exponential link weight distribution

$$
\rho(w)=e^{-w}
$$

is particularly appealing as the node weight distribution in this case is remarkably simple. The governing equations (2) become

$$
(2+w) e^{w} n(w)=\int_{0}^{w} d x x e^{x} n(x)+2 .
$$

Writing $G(w)=2+\int_{0}^{w} d x x e^{x} n(x)$ we recast integral equation (13) into a simple differential equation

$$
\left(\frac{2}{w}+1\right) \frac{d G}{d w}=G
$$

We find $G(w)=8(w+2)^{-2} e^{w}$, from which

$$
n(w)=\frac{1}{\left(1+\frac{w}{2}\right)^{3}} .
$$

Thus for the exponential link weight distribution, the emerging node weight distribution is scale-free (that is, purely algebraic) over the entire weight range.

We now outline the behavior for link distributions with heavy tails. Consider particularly distributions with a power-law tail $\rho(w) \sim w^{-\nu}$; the exponent must obey the inequality $\nu>1$ to ensure normalization $\int d w \rho(w)=1$. It is easy to check that for $\nu>3$ the leading asymptotic of the node weight distribution is $n(w) \sim w^{-3}$; moreover, expansion (III) holds up to the order of $n$, where $n$ is the largest integer smaller than $\nu$ (i.e. $n<\nu<n+1$ ) 25]. For $2<\nu<3$ the leading term shows even slower decay $n(w) \sim(3-\nu)^{-1} w^{-\nu}$.

The situation is very different for $1<\nu \leq 2$ when the total weight grows with size faster than linearly: $W \sim N^{1 /(\nu-1)}$. Using the usual definition $N_{w}=N n(w)$, we observe that the square bracket term in Eq. (1) is negligible for large $N$ values. Hence $n(w) \rightarrow \rho(w)$, suggesting that the number of nodes with more than one links grows slower than linearly in $N$, that is only the dangling nodes give contribution to $n(w)$.

\section{B. Weight-Degree Distribution}

The node weight distribution is perhaps the most natural, and therefore readily tractable, local characteristic for networks with weight-driven growth. Of course, the degree distribution remains geometrically the simplest local characteristic, yet for the weighted networks it is generally impossible to write down a closed equation for the degree distribution $n_{k}$. To compute $n_{k}$, one must determine the joint weight-degree distribution $n_{k}(w)$; the degree distribution is then found by integration

$$
n_{k}=\int_{0}^{\infty} d w n_{k}(w) .
$$

The weight-degree distribution obeys a set of equations similar to (2). The density of dangling nodes is given by

$$
(\lambda+w) n_{1}(w)=\lambda \rho(w)
$$

while for $k \geq 2$ the weight-degree distribution satisfies

$$
(\lambda+w) n_{k}(w)=\int_{0}^{w} d x \rho(w-x) x n_{k-1}(x) .
$$

One can treat Eqs. (17)-(18) recursively, yet even for the simplest link weight distributions like the uniform distribution (3) the exact expressions for $n_{k}(w)$ become very unwieldy as the degree grows. Exceptionally neat results emerge again for the exponential link weight distribution (12). In this case

$$
(2+w) e^{w} n_{k}(w)=\int_{0}^{w} d x e^{x} x n_{k-1}(x) .
$$


Starting with $n_{1}(w)=\frac{2}{2+w} e^{-w}$ we explicitly computed a few more $n_{k}(w)$ which led us to the hypothetical solution (we use the shorthand notation $\Omega=w-2 \ln \left(1+\frac{w}{2}\right)$ )

$$
n_{k}(w)=\frac{1}{\left(1+\frac{w}{2}\right)^{3}} \frac{\Omega^{k-1} e^{-\Omega}}{(k-1) !} .
$$

(Of course, the above ansatz agrees with the sum rule $n(w)=\sum_{k>1} n_{k}(w)$.) Having guessed the solution, it is then straightforward to verify its validity. Note that asymptotically $(k \gg 1)$, the joint weight-degree distribution approaches a Gaussian centered around $\Omega=k-4$, or $w_{k} \approx k+2 \ln k$, with width $\propto \sqrt{k}$. Interestingly, the average weight of the nodes of degree $k$, viz. $w_{k} \approx k+2 \ln k$, slightly exceeds [26] the expected value $w_{k}=k$.

The degree distribution does not admit a simple closed form even for the exponential link weight distribution. The exact expression

$$
n_{k}=\int_{0}^{\infty} \frac{d \Omega}{\frac{w}{2}\left(1+\frac{w}{2}\right)^{2}} \frac{\Omega^{k-1} e^{-\Omega}}{(k-1) !}
$$

simplifies for $k \gg 1$. Indeed, utilizing two properties of the quantity $\Omega^{k} e^{-\Omega} / k$ ! , viz. (i) it has a sharp maximum at $\Omega=k$ and (ii) $\int_{0}^{\infty} d \Omega \Omega^{k} e^{-\Omega} / k !=1$, we estimate the integral in (21) as the value of the slowly varying part of the integrand $\left(\frac{w}{2}\right)^{-1}\left(1+\frac{w}{2}\right)^{-2}$ near the maximum, i.e. at $w_{k} \approx k+2 \ln k$. Thus

$$
n_{k}=\frac{8}{k^{3}}-48 \frac{\ln k}{k^{4}}+\mathcal{O}\left(k^{-4}\right) .
$$

\section{In-Component Weight Distribution}

The emerging network has the natural structure of the directed graph since each new link starts at the new node and ends up at some previous node. Taking into account the orientation of each link allows to define an incomponent and an out-component with respect to each node. For instance, the in-component of node $\mathbf{x}$ is the set of all nodes from which node $\mathbf{x}$ can be reached following a path of directed links (Fig. 22). The computation of in- and out-component size distributions is quite complicated as one must first determine joint distributions that involve both size and weight. In contrast, in- and out-component weight distributions can be determined directly. Here we compute the in-component weight distribution.

Let $w_{0}$ be the weight of the link emanating from node $\mathbf{x}$ and $w$ the total weight of all other links in the incomponent of node $\mathbf{x}$; then the total weight of that incomponent, that is the sum of weights of all nodes in in-component of node $\mathbf{x}$, is $w_{0}+2 w$. Denote by $i\left(w_{0}, w\right)$ the density of such in-components. Here we tacitly assume that $w>0$, that is the size of the in-components is larger than one. The density $i\left(w_{0}\right)$ of in-components of size one and weight $w_{0}$ is found by noting that such

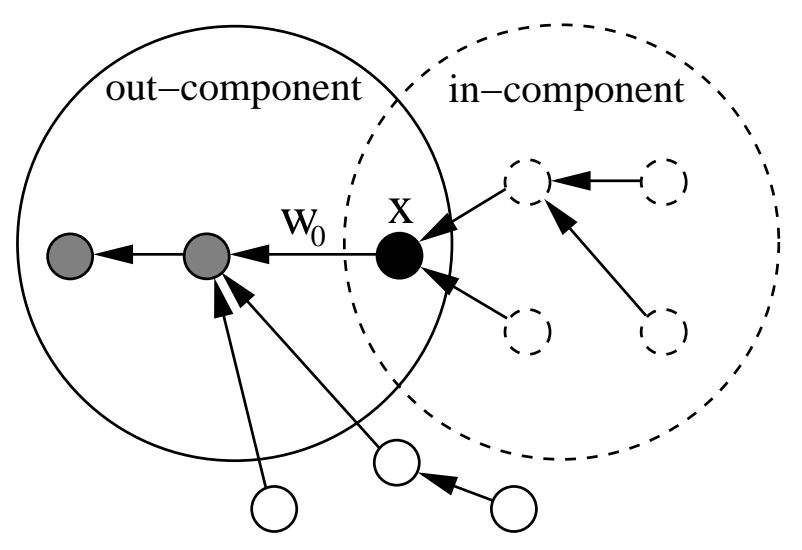

FIG. 2: In and out components of node $\mathbf{x}$. In this example, the out-component has size 3 and the in-component has size 5 (node $\mathbf{x}$ itself belongs to its in and out components).

in-components are just dangling nodes, so

$$
i\left(w_{0}\right) \equiv n_{1}\left(w_{0}\right)=\frac{\lambda}{\lambda+w_{0}} \rho\left(w_{0}\right) .
$$

The density $i\left(w_{0}, w\right)$ satisfies

$$
\begin{aligned}
\lambda i\left(w_{0}, w\right) & =\int_{0}^{w} d x \rho(w-x)\left(w_{0}+2 x\right) i\left(w_{0}, x\right) \\
& -\left(w_{0}+2 w\right) i\left(w_{0}, w\right)+i\left(w_{0}\right) w_{0} \rho(w) .
\end{aligned}
$$

For the exponential link weight distribution (12), this equation can be re-written in a simple form

$$
\left(\frac{2}{w_{0}+2 w}+1\right) \frac{\partial G}{\partial w}=G
$$

where $G=G\left(w_{0}, w\right)$ is the auxiliary variable

$$
G=\int_{0}^{w} d x e^{x}\left(w_{0}+2 x\right) i\left(w_{0}, x\right)+\frac{2 w_{0}}{2+w_{0}} e^{-w_{0}} .
$$

Solving (24) we obtain $G\left(w_{0}, w\right)=G\left(w_{0}, 0\right) \frac{2+w_{0}}{2+w_{0}+2 w} e^{w}$. Equation (25) gives the initial condition, so the auxiliary variable reads $G=\frac{2 w_{0}}{2+w_{0}+2 w} e^{w-w_{0}}$. This result leads to the in-component density

$$
i\left(w_{0}, w\right)=\frac{2 w_{0}}{\left(2+w_{0}+2 w\right)^{2}} e^{-w_{0}} .
$$

Now the total weight of the in-component is $s=w_{0}+2 w$, and the respective weight distribution $I(s)$ is

$$
I(s)=i(s)+\frac{1}{2} \int_{0}^{s} d w_{0} i\left[w_{0},\left(s-w_{0}\right) / 2\right] .
$$

Plugging (23) and (26) into (27) we arrive at

$$
I(s)=\frac{1}{(2+s)^{2}}+\left[\frac{1}{2+s}+\frac{1}{(2+s)^{2}}\right] e^{-s},
$$

Therefore up to an exponentially small correction, the incomponent weight distribution is algebraic with exponent 2 (the same exponent characterizes the in-component size distribution [27], which is not so surprising as this exponent is found to be very robust). 


\section{GENERALIZATIONS}

The model of the previous section always results in a tree structure by construction. In this section we consider two generalizations of that model which allows the formation of loops in the evolving network.

The simplest generalization of the minimal model leading to a network with many loops is to connect a newly created node to $m$ target nodes. The attachment probability is still proportional to the weight of the target node as before, and the weights of the $m$ new links are chosen independently from the link weight distribution $\rho(w)$. The weight of the newly introduced node becomes the sum of the $m$ independent link weights, which is then distributed according to $\rho_{m}(w)$, the $m$-fold convolution of $\rho(w)$. The governing equation thus remains similar to Eq. (1) except that the last term $\rho(w)$ becomes $\rho_{m}(w)$, and the square bracketed term gains a factor $m$ due to the $m$ attached links. As the average node weight is also $m$ times larger than previously, that is $\lambda=2 m\langle w\rangle$, and the node density satisfies equation

$$
(2\langle w\rangle+w) n(w)=\int_{0}^{w} d x \rho(w-x) x n(x)+2\langle w\rangle \rho_{m}(w)
$$

which is almost identical to Eq. (2), the only difference is the second term on the right-hand side which now contains $\rho_{m}(w)$ instead of $\rho(w)$. The modified model leads to the same $\sim w^{-3}$ tail for the node weight distribution for any $\rho(w)$ with a cutoff since then $\rho_{m}(w)$ also has a cutoff and the same argument applies as before. For the exponential link weight distribution $\rho(w)=e^{-w}$ the convoluted distribution is $\rho_{m}(w)=e^{-w} w^{m-1} /(m-1)$ !, and the resulting node weight distribution is algebraic (up to an exponentially small correction)

$$
n(w) \rightarrow \frac{2 m(m+3)}{(2+w)^{3}} \quad \text { for } \quad w \gg 1 .
$$

A more substantial generalization of the minimal model is to allow the creation of links between already existing nodes [28]. We again choose the simplest weightdriven rule, namely we assume that a new link is created with rate proportional to the product of weights of the originating and target nodes. Let $r$ be the average number of link creations between already existing nodes per node creation, so in the network with $N$ nodes the average number of links is $(1+r) N$. This can be modeled by two independent processes: with probability $1 /(1+r)$, a new node is attached to the network, and with probability $r /(1+r)$ a new link is added between two already existing nodes. The weight distribution $N_{w}(N)$ satisfies an equation similar to (11), with the term in the square brackets multiplied by $(1+2 r) / W$. The governing equation for the normalized weight distribution $n(w)$ is therefore almost identical to Eq. (2), the only change is the replacement $\lambda \rightarrow \Lambda=\lambda /(1+2 r)$ :

$$
(\Lambda+w) n(w)=\int_{0}^{w} d x \rho(w-x) x n(x)+\Lambda \rho(w) .
$$

The average node weight $\lambda=\int_{0}^{\infty} d w w n(w)$ is now equal to $2(1+r)\langle w\rangle$ since the number of links now $1+r$ times exceeds the number of nodes and each link contributes twice. Multiplying (29) by $w$ and integrating over $w$ we indeed obtain $\lambda=2(1+r) \int_{0}^{\infty} d w w \rho(w)$ thereby providing a useful check of self-consistency.

For the exponential link weight distribution (12) the node weight distribution satisfies

$$
\left(1+\frac{1+2 r}{2+2 r} w\right) e^{w} n(w)=1+\frac{1+2 r}{2+2 r} \int_{0}^{w} d x x e^{x} n(x)
$$

Solving this equation we again obtain the purely algebraic node weight distribution:

$$
n(w)=\left(1+\frac{1+2 r}{2+2 r} w\right)^{-\nu}, \quad \nu=\frac{3+4 r}{1+2 r}
$$

The exponent $\nu$ monotonously decreases from 3 to 2 as $r$ increases from 0 to $\infty$.

\section{CONCLUSIONS}

We examined a minimal model for the weight-driven network growth. The virtue of the minimal model is that its many features like the node weight distribution, the joint node weight-degree distribution, the in-component weight distribution, etc. are tractable analytically. In particular, we showed that the node weight distribution exhibits a universal $w^{-3}$ tail independently on the link weight distribution (as long as the tail of the latter is sharper than $w^{-3}$ ), and the in-component weight distribution displays a robust $s^{-2}$ tail.

Remarkably simple behaviors characterize the exponential link weight distribution: The emerging node weight distribution is purely algebraic, the joint node weight-degree distribution and the in-component weight distribution are also given by neat closed formulas.

We also studied generalizations of the minimal model for the weight-driven network growth. When a new node is connected to several target nodes and/or links are additionally created between already existing nodes, the network acquires loops yet it remains tractable. One generic feature of this class of models is that the node weight distribution remains algebraic, $n(w) \sim w^{-\nu}$, with the exponent $\nu$ varying from 3 to 2 as the average node degree increases from one to $\infty$.

\section{ACKNOWLEDGMENT}

TA thanks the Swiss NSF for financial support under the fellowship 8220-067591. 
[1] S. N. Dorogovtsev and J. F. F. Mendes, Evolution of networks: From Biological Nets to the Internet and $W W W$ (Oxford University Press, Oxford, 2003); R. PastorSatorras and A. Vespignani, Evolution and Structure of the Internet: A Statistical Physics Approach (Cambridge University Press, Cambridge, 2004).

[2] P. Erdős and P. Rényi, Publ. Math. Inst. Hung. Acad. Sci. 5, 17 (1960).

[3] B. Bollobás, Random Graphs (Academic Press, London, 1985).

[4] R. Albert and A.-L. Barabási, Rev. Mod. Phys. 74, 47 (2002).

[5] P. L. Krapivsky and S. Redner, Computer Networks 39, 277 (2002).

[6] M. E. J. Newman, SIAM Review 45, 167 (2003).

[7] M. E. J. Newman, Phys. Rev. E 64, 016131 (2001); ibid, 64, 016132 (2001).

[8] A.-L. Barabási, H. Jeong, Z. Neda, E. Ravasz, A. Schubert, and T. Vicsek, Physica A 311, 590 (2002).

[9] R. Guimera, M. Sales-Pardo, and L. A. N. Amaral, cond-mat/0312535

[10] A. Barrat, M. Berthelemy, and A. Vespignani, Proc. Natl. Acad. Sci. 101, 3747 (2004); Phys. Rev. Lett. 92, 228701 (2004).

[11] L. R. Ford and D. R. Fulkerson, Flows in Networks (Princeton University Press, Princeton, N.J., 1962)

[12] R. K. Ahuja, T. L. Magnanti, and J. B. Orlin, Network Flows: Theory, Algorithms, and Applications (Prentice Hall, Englewood Cliffs, N.J., 1993).

[13] P. G. Doyle and J. L. Snell, Random Walks and Electric Networks (Math. Assoc. Amer., 1984).

[14] L. de Arcangelis, S. Redner, and A. Coniglio, Phys. Rev. B 31, 4725 (1985); Phys. Rev. B 34, 4656 (1986).

[15] R. Rammal, C. Tannous, P. Breton and A.-M. S. Tremblay, Phys. Rev. Lett. 54, 1718 (1985).

[16] M. E. J. Newman, cond-mat/0407503
[17] E. Almaas, P. L. Krapivsky, and S. Redner, condmat/0408xxx.

[18] S. H. Yook, H. Jeong, A.-L. Barabási, and Y. Tu, Phys. Rev. Lett. 86, 5835 (2001).

[19] J. D. Noh and H. Rieger, Phys. Rev. E 66, 066127 (2002).

[20] D. Zheng, S. Trimper, B. Zheng, and P. M. Hui, Phys. Rev. E 67, 040102(R) (2003).

[21] P. J. Macdonald, E. Almaas, and A.-L. Barabási, cond-mat/0405688

[22] A. Barrat, M. Berthelemy, and A. Vespignani, cond-mat/0406238

[23] Negative weights are occasionally appropriate, e.g. they can represent animosity between individuals in a social network.

[24] $N$ is a discrete variable and $N_{w}(N)$ are random variables. Treating $N$ as a continuous variable and $N_{w}(N)$ as the average values of the corresponding random variables is asymptotically exact when the weight is sufficiently small; see e.g. P. L. Krapivsky and S. Redner, J. Phys. A 35, 9517 (2002) for the detailed analysis of these issues in the model where growth is governed by preferential attachment.

[25] For integer $\nu \geq 4$, the $\nu^{\text {th }}$ term in expansion (11) acquires a logarithmic correction; for $\nu=3$, even the leading order term has a logarithmic correction $n(w) \sim w^{-3} \ln (w)$.

[26] The expected value for the sum of $k$ independent identically distributed random variables taken from the exponential distribution is $w=k$; in the present case the average weight of the node of large degree is (slightly) higher since the growth is weight-driven.

[27] P. L. Krapivsky and S. Redner, Phys. Rev. E 63, 066123 (2001).

[28] P. L. Krapivsky, G. J. Rodgers, and S. Redner, Phys. Rev. Lett. 86, 5401 (2001). 